\title{
Can Routine Blood Biochemistry Parameters be Predictive Prognostic Marker(s) in Operated Patients with Meningioma WHO Grade 1?
}

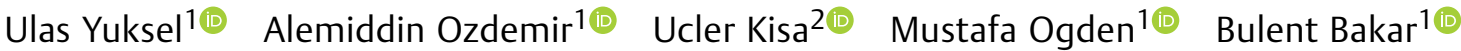 \\ ${ }^{1}$ Department of Neurosurgery, Kirikkale University Faculty of \\ Medicine, Kirikkale, Turkey \\ 2 Department of Biochemistry, Kirikkale University Faculty of \\ Address for correspondence Bulent Bakar, MD, Department of \\ Neurosurgery, Kirikkale University Faculty of Medicine, Yahsihan \\ Yerleskesi, 71450-Kirikkale, Turkey \\ Medicine, Kirikkale, Turkey \\ (e-mail: bulentbanrs@yahoo.com).
}

Arq Bras Neurocir 2021;40(2):e137-e145.

\begin{abstract}
Keywords

- biochemistry

- biomarker

- meningioma

- prognosis

Background Today, there is a need for new and independent additional advanced markers that can predict the prognosis of meningioma patients, postoperatively. The present study aimed to find out postoperative short-term prognostic markers in patients with meningioma using their demographic data and routine blood biochemistry findings evaluated preoperatively.

Methods The Glasgow Coma Scale (GCS), and Glasgow Outcome Scale (GOS) scores of the patients were recorded. Additionally, preoperatively obtained serum glucose, Creactive protein (CRP), sodium, potassium, creatinine, blood urea nitrogen, aspartate aminotransferase (AST), alanine aminotransferase, and hemoglobin level values, platelet, leukocyte, neutrophil, lymphocyte, eosinophil, basophil, and monocyte count results, erythrocyte sedimentation rate (ESR), neutrophil-lymphocyte ratio, plateletlymphocyte ratio (PLR) and lymphocyte-monocyte ratio (LMR) values were evaluated. Results In the present study, 23 operated patients with meningioma World Health Organization (WHO) grade 1 (17 females, 6 males) were included. Correlation test results revealed that the GCS score, platelet count, and serum potassium level values could directly predict the short-term prognosis of these patients. Additionally, these test results suggested that the lymphocyte, monocyte, and eosinophil count values, PLR, LMR, ESR, serum glucose, CRP, and AST level values could be indirect markers in predicting the short-term prognosis. However, likelihood ratio test results revealed that only monocyte count value, LMR value, and serum CRP level value could be the markers for prediction of the short-term prognosis.

Conclusion At the end of the present study, it was concluded that the monocyte count value, LMR value, and serum CRP level value could be the best markers in predicting the short-term prognosis of the operated meningioma patients.
\end{abstract}

received

August 8, 2020

accepted

November 16, 2020

published online

January 18, 2021
DOI https://doi.org/

10.1055/s-0040-1722246. ISSN 0103-5355.

\footnotetext{
(c) 2021. Sociedade Brasileira de Neurocirurgia. All rights reserved. This is an open access article published by Thieme under the terms of the Creative Commons Attribution-NonDerivative-NonCommercial-License, permitting copying and reproduction so long as the original work is given appropriate credit. Contents may not be used for commercial purposes, or adapted, remixed, transformed or built upon. (https://creativecommons.org/ licenses/by-nc-nd/4.0/)

Thieme Revinter Publicações Ltda., Rua do Matoso 170, Rio de Janeiro, RJ, CEP 20270-135, Brazil
} 


\section{Introduction}

It has been shown that many immunological agents produced in tumor tissue in many types of cancer can stimulate the bone marrow to increase myelopoiesis and subsequently lead to an increase in circulating granulocytes. ${ }^{1}$ Most tumors have been reported to be highly infiltrated by immune cells, including macrophages, neutrophils, and lymphocytes. ${ }^{2}$ This systemic inflammation is a product of circulating immune cells and cytokines. ${ }^{3}$ To be able to classify and predict survival, there has recently been a great deal of research on molecular markers derived from genetic and epigenetic studies on tumor tissue. ${ }^{4}$

Meningiomas, slow-growing brain tumors, can be still treated with total resection surgery. However, the prognosis of the patients with benign meningioma treated surgically has been reported to be worse than in the general population. ${ }^{5}$ Previous reports in the literature have stated that interleukin-6 (IL-6) plays a role in meningioma cell proliferation and growth, and even meningioma cells produce and secrete this cytokine. Additionally, IL-6 mRNA and protein expression have been reported to be positively correlated with peritumoral edema around the meningioma. ${ }^{6,7}$ Unfortunately, most of these markers were investigated in the tumor tissue and, therefore, it is considered to exist still a need for new additional independent biomarkers that can be inexpensive, noninvasive and easily applicable in any medical laboratory for prediction of prognosis in patients with meningioma, preoperatively. ${ }^{8}$ The present study aimed to find out the postoperative shortterm prognostic markers in patients with meningioma by using demographic, neurological status, and routine blood biochemistry findings, evaluated preoperatively.

\section{Materials and Methods}

\section{Patient Groups}

Approval for the present retrospective single-center study was granted by the Clinical Studies Local Ethics Committee (approval date: April 29th, 2020, and approval number: 2020.04.06).

A retrospective evaluation was made of patients with solitary intracranial tumors who were admitted to the Neurosurgery Clinic between January 2015 and April 2020. Then, operated patients whose histopathological diagnosis was typical meningioma World Health Organization (WHO) grade 1 were included in the present study. To investigate the effects of the age of the patients on the data of the patients, the patients were divided into two groups according to age, as follows:

- ADULT group (consisted of $<65$-year-old patients, $n=13$ )

- ELDERLYgroup (consisted of $\geq 65$-year-old patients, $n=10$ )

Moreover, to investigate the effects of gender on the data of the patients, the patients were divided into two groups according to gender.

- FEMALE group $(n=17)$

- MALE group $(n=6)$

Patients with atypical or malignant meningioma, patients with other types of intracranial tumors such as glioblastoma, patients treated with recurrence of intracranial tumors, patients treated with radiosurgery, patients with multiple intracranial meningiomas, and pediatric patients $(<16$ years old) were excluded from the present study.

\section{Methods}

Age, gender, and routine blood biochemistry results of all participants were recorded. Additionally, the Glasgow Coma Scale (GCS) scores, duration of stay in hospital, and Glasgow Outcome Scale (GOS) scores of the patients were recorded. In the present study, the consciousness level and neurological condition of the patients at admission to the hospital were determined using the GCS. ${ }^{9}$ The short-term prognosis was determined based on the neurological condition of the patient at discharge from the hospital after surgical treatment. This neurological condition was considered using the GOS, which was accepted in the literature as a scale to demonstrate the short-term prognosis of any patient. ${ }^{10}$ Because the present study was planned to determine mainly the short-term prognosis of the patients after surgical treatment, the long-term follow-up findings of the patients was not included in the present study.

\section{Biochemical Analysis}

Venous blood samples collected preoperatively from the patients with meningioma were examined in the biochemistry laboratory. Serum glucose (reference interval 4.11-6.05 $\mathrm{mmol} / \mathrm{L}$ ), C-reactive protein (CRP) (reference interval 1.5-50.00 $\mathrm{mg} / \mathrm{L}$ ), creatinine (reference interval 74.26-109.62 $\mu \mathrm{mol} / \mathrm{L}$ ), blood urea nitrogen (BUN) (reference interval 6.07-15.35 $\mathrm{mmol} / \mathrm{L}$ ), aspartate aminotransferase (AST) (reference interval 0.08-0.67 $\mu \mathrm{kat} / \mathrm{L}$ ) and alanine aminotransferase (ALT) (reference interval $0.08-0.68 \mu \mathrm{kat} / \mathrm{L}$ ) levels were measured using the immunoturbidimetric method. ${ }^{11}$ Serum sodium (reference $136-146 \mathrm{mmol} / \mathrm{L}$ ) and potassium (reference interval 3.5-5.1 $\mathrm{mmol} / \mathrm{L}$ ) level values were determined with the ion-selective electrode (ISE) method. ${ }^{12}$ All parameters were studied on an analyzer (COBAS c501, Roche Diagnostics, Basel, Switzerland) using their original commercial kits (Roche, Basel, Switzerland).

Blood hemoglobin level values (reference interval $100.00-180.00 \mathrm{~g} / \mathrm{L}$ ) and platelet (reference interval 150.00$500.00 \wedge 109 / \mathrm{L}$ ), leukocyte (reference interval 4.40-11.30 $\wedge 109 / \mathrm{L}$ ), neutrophil (reference interval 1.10-9.60^109/L), lymphocyte (reference interval 500-6,000/uL), eosinophil (reference interval $0.50-6.0 \wedge^{\wedge} 109 / \mathrm{L}$ ), eosinophil (reference interval $0-1.00^{\wedge} 109 / \mathrm{L}$ ), basophil (reference interval $0-0.30$ $\wedge 109 / \mathrm{L}$ ) and monocyte (reference interval 0.10-1.40^109/L) count results were determined using an analyzer (Mindray BC-6800, Shenzen, China). The erythrocyte sedimentation rate (ESR) value (reference range $<20 \mathrm{~mm} /$ hour) was measured using an automated system (ESR 40, Sistat Diagnostics, Ankara, Turkey). The neutrophil to lymphocyte ratio (NLR), platelet to lymphocyte ratio (PLR), lymphocyte to monocyte ratio (LMR), and systemic inflammation response index (SIRI) were also calculated as defined in the literature. ${ }^{11-13}$

\section{Statistical Analysis}

Parametric data were expressed as mean \pm standard deviation (SD), and to find out the differences between the study groups, 
these data were analyzed using the independent samples t-test $(p<0.05)$. Nonparametric data were expressed as median (minimum-maximum), and to find out the differences between the study groups, these data were analyzed using the Mann Whitney U-test $(p<0.05)$. Categorical data were expressed as number (\%), and to find out the differences between the study groups, they were analyzed using the Pearson chi-squared test $(p<0.05)$.

To determine which parameters could relate to the prognosis of the patients, the correlations between the study parameters and GOS scores were analyzed using the Spearman rho correlation test $(p<0.05)$. To identify the best marker(s) in predicting the short-term prognosis, the likelihood-ratio test was applied to the variables that were found in a relationship with the prognosis $(p<0.05)$.

\section{Results}

The study group comprised 23 patients (17 females, 6 males) with a mean age of $61.39 \pm 12.06$ years old, the median GCS score was 15 points ( 10 points in 1 patient, 15 points in 22 patients), and the median GOS score was 5 points. One patient died due to massive thromboembolism, and one patient died after acute massive bleeding in the postoperative surgical field (-Fig. 1). One patient with a GOS score of 3 points had dementia. One of the two patients with a GOS score of 4 points had left hemiplegia, postoperatively (-Fig. 2), and the other one had right hemiparesis, postoperatively (-Table $\mathbf{1}$ ).

On the other hand, when the patients were divided into two groups according to age, it was seen that serum CRP level value $(Z=-1.243, p=0.035)$ and the duration of stay in hospital $(Z=-2.099, p=0.036)$ were found to be higher in patients of the ELDERLY group. However, the serum CRP levels of both groups were observed in the laboratorial normal reference range (-Table 2 ).

Additionally, when the patients were divided into two groups according to gender, it was observed that hemoglobin level values $(t=-3.864, p=0.001)$, serum creatinine level values $(t=-3.386, p=0.003)$, and ESR values $(t=3.909$, $p=0.001$ ) were found to be different between female and male patients. It was argued that hemoglobin and serum creatinine levels were higher in male patients, whereas ESR levels were lower. However, except for the ESR values, the serum hemoglobin and serum creatinine levels were observed in the laboratorial normal reference range in both groups (-Table $\mathbf{3}$ ).

The results of the Spearman rho correlation test, applied to all parameters of all patients, revealed that there was a positive correlation between age and serum CRP level values $(r=0.424, p=0.044)$, and between age and duration of stay in hospital ( $r=0.547, p=0.007)$. A positive correlation was observed between gender and hemoglobin level $(r=0.627$, $p=0.001$ ), and between gender and serum creatinine level $(r=0.538, p=0.008)$. The GCS scores correlated positively with the GOS scores $(r=0.529, p=0.009)$, basophil count values $(r=0.466, p=0.025)$, and platelet count values $(r=0.467, p=0.018)$. A positive correlation was found between GOS scores and platelet count values $(r=0.511$, $p=0.013$ ), between the GOS scores and serum potassium level values $(r=0.508, p=0.013)$, and between hemoglobin and serum creatinine level values $(r=0.560, p=0.005)$. The leukocyte count values correlated positively with the neutrophil count values $(\mathrm{r}=0.958, p<0.001)$, NLR values $(\mathrm{r}=0.619, p=0.002)$, and serum creatinine level values
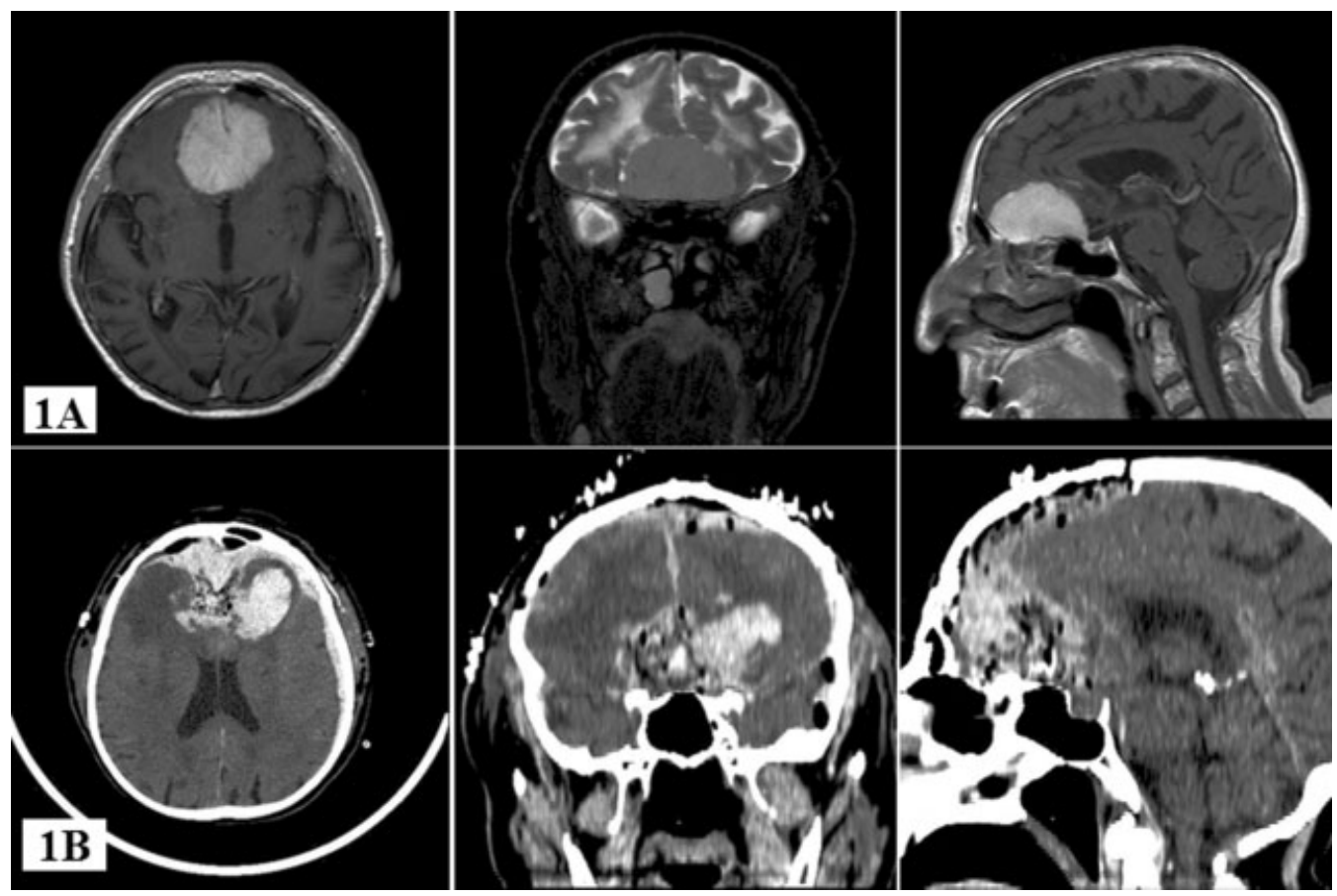

Fig. 1 Preoperative magnetic resonance imaging (MRI) with gadolinium T1-weighted images show the giant olfactory groove meningioma of the patient who died during the stay in the hospital (1A); and early postoperative computed tomography images show that the tumor was totally removed, but postoperative acute massive blood filled in the surgical field (1B). 

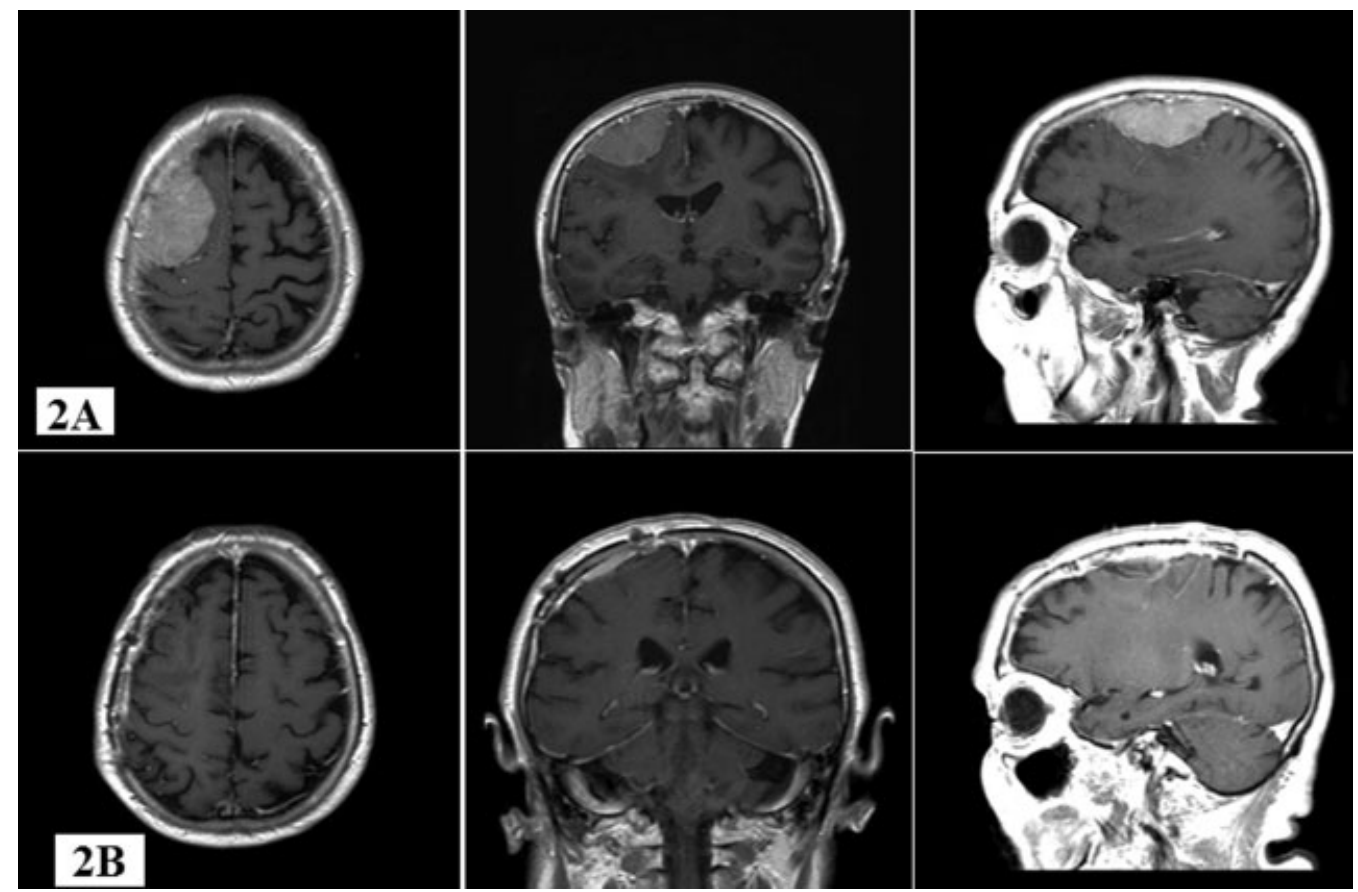

Fig. 2 Preoperative magnetic resonance imaging (MRI) with gadolinium T1-weighted images show the parasagittal meningioma of the patient whose Glasgow Outcome Score was 5 points (2A), and early postoperative images show that the tumor was totally removed (2B).

$(\mathrm{r}=0.445, p=0.033)$. The neutrophil count values correlated positively with the NLR values $(r=0.776, p<0.001)$, serum creatinine level values $(r=0.531, p=0.009)$, and serum BUN level values $(r=0.428, p=0.042)$. A positive correlation was found between the lymphocyte and monocyte count values $(r=0.533, p=0.009)$, between the LMR and SIRI values $(\mathrm{r}=0.825, p<0.001)$, between LMR and serum sodium values $(r=0.532, p=0.009)$, between LMR and serum AST values $(r=0.532, p=0.010)$, between eosinophil count and basophil count values $(r=0.542, p=0.008)$, between basophil count and platelet count values $(r=0.417, p=0.048)$, between NLR and serum BUN level values $(r=0.455$, $p=0.029$ ), between NLR and serum creatinine level values $(\mathrm{r}=0.545, p=0.007)$, between PLR and serum AST level values $(\mathrm{r}=0.451, p=0.031)$, between PLR and ESR values $(\mathrm{r}=0.426, p=0.043)$, between serum glucose level values and duration of stay in hospital $(r=0.462, p=0.027)$ and between serum CRP level values and duration of stay in hospital ( $\mathrm{r}=0.591, p=0.003)$.

On the other hand, a negative correlation was determined between age and GCS score values $(r=-0.452, p=0.030)$, between age and serum potassium level values $(r=-0.450$, $p=0.031)$, between gender and ESR values $(r=-0.650$, $p=0.001$ ), between hemoglobin level values and ESR values $(r=-0.571, p=0.004)$, between lymphocyte count values and NLR values $(r=-0.679, p<0.001)$, between lymphocyte count values and PLR values $(r=-0.900, p<0.001)$ and between eosinophil count values and PLR values $(r=-$ $0.422, p=0.045$ ).

Although the monocyte count values, LMR values, and CRP level values did not correlate with the GOS score values, the likelihood-ratio test results revealed that monocyte count value $\left(X^{2}=10.595, p=0.014\right)$, LMR value $\left(X^{2}=8.682, p=0.034\right)$, and serum CRP level value $\left(\mathrm{X}^{2}=8.028, p=0.045\right)$ could be the best parameters for prediction of the short-term prognosis of these patients.

\section{Discussion}

In the literature, it has been suggested that serum CRP levels, which reflect the systemic response to inflammation, are related to progression of the cancer, and elevated CRP levels may be associated with poor prognosis. ${ }^{8,13-16}$ In those studies, it has been concluded that the CRP level may be a biomarker for many cancer types. ${ }^{17,18}$ However, in their literature review, Heikkilä et al. found no strong evidence for a causal role of serum CRP level in malignancy. ${ }^{19}$ On the other hand, Bunevicius et al. reported in their study that there may be a relationship between high CRP concentration and shorter survival in meningioma patients, and at the end of this study, they suggested that meningioma patients presenting with high CRP levels could be considered with a high risk of complications. ${ }^{6}$

Additionally, it is suggested that a higher number of circulating neutrophils, monocytes, or platelets may be associated with increased tumor progression and poor survival in many types of cancer. ${ }^{20-24}$ Moreover, Liang et al. discussed in their article that meningiomas are infiltrated with lymphocytes, macrophages, mast cells, monocytes, and microglia. They suggested that monocytes can directly stimulate tumor cell growth producing various proinflammatory cytokines such as tumor necrosis factor, IL-6, and IL-1. Tumor-associated macrophages can also increase tumor progression, and the number of circulating monocytes may reflect the formation or presence of tumor-associated macrophages. On the other hand, lymphocytes can migrate into the tumor to create a defense barrier 
Table 1 Demographic and laboratory findings of the patients

\begin{tabular}{|c|c|c|}
\hline Variable & & $\begin{array}{l}\text { Mean } \pm S D / \text { Median } \\
(\min -\max ) / \mathrm{N}(\%)\end{array}$ \\
\hline Age (years old) & & $61.39 \pm 12.06$ \\
\hline \multirow[t]{2}{*}{ Gender } & Female & 17 (39.5\%) \\
\hline & Male & $6(14.0 \%)$ \\
\hline Glasgow Coma Scale score & & $15(10-15)$ \\
\hline $\begin{array}{l}\text { Duration of stay in } \\
\text { hospital (days) }\end{array}$ & & $10(6-51)$ \\
\hline $\begin{array}{l}\text { Glasgow Outcome } \\
\text { Scale score }\end{array}$ & & $5(1-5)$ \\
\hline Hemoglobin level (g/L) & & $133.30 \pm 2.01$ \\
\hline Leukocyte count $\left(\wedge 10^{9} / \mathrm{L}\right)$ & & $9.09 \pm 3569.83$ \\
\hline Neutrophil count $\left(\wedge 10^{9} / \mathrm{L}\right)$ & & $6.44 \pm 3611.59$ \\
\hline Lymphocyte count $\left(\wedge 10^{9} / \mathrm{L}\right)$ & & $2.07 \pm 1126.40$ \\
\hline Monocyte count $\left(\wedge 10^{9} / \mathrm{L}\right)$ & & $0.42 \pm 195.10$ \\
\hline Eosinophil count $\left(\wedge 10^{9} / \mathrm{L}\right)$ & & $0.14 \pm 116.52$ \\
\hline Basophil count $\left(\wedge 10^{9} / \mathrm{L}\right)$ & & $0.04 \pm 40.70$ \\
\hline Platelet count (^109/L) & & $262.13 \pm 68194.44$ \\
\hline $\begin{array}{l}\text { Systemic inflammation } \\
\text { response index }\end{array}$ & & $0.75(0.37-17.89)$ \\
\hline $\begin{array}{l}\text { Neutrophil to } \\
\text { lymphocyte ratio }\end{array}$ & & $2.56(1.10-33.14)$ \\
\hline $\begin{array}{l}\text { Platelet to } \\
\text { lymphocyte ratio }\end{array}$ & & $\begin{array}{l}126.33 \\
(33.17-454.55)\end{array}$ \\
\hline $\begin{array}{l}\text { Lymphocyte to } \\
\text { monocyte ratio }\end{array}$ & & $5.32(0.69-22)$ \\
\hline Glucose (mmol/L) & & $6.05(4.44-15.26)$ \\
\hline Sodium (mmol/L) & & $141(138-148)$ \\
\hline Potassium (mmol/L) & & $4.48 \pm 0.56$ \\
\hline Creatinin $(\mu \mathrm{mol} / \mathrm{L})$ & & $63.65 \pm 0.17$ \\
\hline $\begin{array}{l}\text { Blood urea nitrogen } \\
(\mathrm{mmol} / \mathrm{L})\end{array}$ & & $10.97 \pm 8.65$ \\
\hline $\begin{array}{l}\text { Aspartate } \\
\text { aminotransferase } \\
(\mu \mathrm{kat} / \mathrm{L})\end{array}$ & & $0.31 \pm 6.66$ \\
\hline $\begin{array}{l}\text { Alanin } \\
\text { aminotransferase } \\
(\mu \mathrm{kat} / \mathrm{L})\end{array}$ & & $0.32(0.12-1.50)$ \\
\hline $\begin{array}{l}\text { C-reactive protein } \\
(\mathrm{mg} / \mathrm{L})\end{array}$ & & $26.70(6.90-830.00)$ \\
\hline $\begin{array}{l}\text { Erythrocyte } \\
\text { sedimentation } \\
\text { rate (mm/hour) }\end{array}$ & & $20.13 \pm 11.82$ \\
\hline
\end{tabular}

Abbreviations: max, maximum; min, minimum; $\mathrm{N}$, number of patients; SD, standard deviation.

against tumor cell invasion. Therefore, low lymphocyte count in peripheral blood can cause weak defenses against tumors. At the end of their study, they demonstrated that a low LMR was associated with high tumor grade. ${ }^{13,25}$

In the present study, all data of the patients revealed that most of the patients were female patients $<65$ years old. The inflammatory cell count results were found to be within normal intervals. The serum glucose levels were at the upper limit of normal laboratory values and the creatinine levels were at the lower limit of normal laboratory values in all patients. On the other hand, ESR values $(20.13 \pm 11.82$ $\mathrm{mm}$ /hour) were at the upper limit of normal, as described by Strojnik et al., ${ }^{8}$ whereas the serum CRP levels $(26.70 \mathrm{mg} / \mathrm{L})$ were found within the normal reference interval ( - Table 1 ).

When the patients were divided into two groups as "adult" and "elderly" patients, it was seen that the elderly patients (age $>65$ years old) stayed longer in the hospital, and that their serum CRP level values were slightly higher than those of adult patients, but within a normal laboratory reference range (-Table $\mathbf{2}$ ). On the other hand, when the patients were divided into two groups according to gender, hemoglobin level values of the female patients were lower than those of male patients, but within a normal laboratory reference range, whereas their ESR values were higher than the values of male patients ( - Table 3 ).

The results of the Spearman rho correlation test results performed on the data of all revealed that if the age of the patients increased, the GCS scores might be low, and serum potassium values might be measured at the lower limit of normal laboratory values. Additionally, in these patients, the duration of stay in the hospital might be longer, and CRP values might be at the upper limit of normal laboratory values. On the other hand, if the GCS scores of the patients were high, it was considered that GOS scores, basophil, eosinophil, and platelet count values could also be high. Moreover, if the platelet count and serum potassium level values were found at the upper limit of normal laboratory values, it was thought that these patients would have higher GOS scores. Briefly, it was assumed, according to the correlation test results, that the GCS scores and GOS scores of elderly patients may be lower than those of adult patients. Therefore, it was thought that elderly patients with meningioma may have a worse neurological condition on admission to the hospital, and that they would have a poor prognosis during the discharge from the hospital, postoperatively. In conclusion, it was considered that GCS score, platelet count, and serum potassium level values could predict directly the short-term prognosis of meningioma patients. In addition, it was argued that age, serum CRP level values, duration of stay in the hospital, eosinophil, and basophil count values could indirectly predict the short-term prognosis of these patients.

Additionally, the correlation test results revealed that if the platelet and basophil count values were high, these patients could have high GCS scores, and, indirectly, they could have high GOS scores and high eosinophil count values. On the other hand, the PLR values correlated positively with the serum AST and ESR values and correlated negatively with eosinophil and lymphocyte count values. Serum AST level values were positively correlated with LMR and SIRI values. The lymphocyte count values were positively correlated with monocyte count values. Therefore, if any of the lymphocyte, eosinophil, or monocyte count values were low, it was predicted that the PLR value and, therefore, LMR, SIRI, ESR, and serum AST level 
Table 2 Demographic findings and laboratory results of the ADULT and ELDERLY groups

\begin{tabular}{|c|c|c|c|c|c|}
\hline & & ADULT $(n=13)$ & ELDERLY $(n=10)$ & & \\
\hline Variable & & $\begin{array}{l}\text { Mean } \pm S D / \text { Median } \\
(\min -\mathrm{max}) / \mathrm{N}(\%)\end{array}$ & $\begin{array}{l}\text { Mean } \pm \text { SD/Median } \\
(\min -\max ) / \mathrm{N}(\%)\end{array}$ & $t / Z \mid X^{2}$ & p-value \\
\hline Age (years old) & & $53.38 \pm 8.27$ & $71.80 \pm 7.14$ & - & - \\
\hline \multirow[t]{2}{*}{ Gender } & Female & $11(47.8 \%)$ & $6(26.1 \%)$ & $1.776^{\ddagger}$ & 0.183 \\
\hline & Male & $2(8.7 \%)$ & $4(17.4 \%)$ & & \\
\hline Glasgow Coma Scale & & $15(15-15)$ & $15(10-15)$ & $-1.649^{\dagger}$ & 0.099 \\
\hline Duration of hospital stay (days) & & $8(6-31)$ & $11.5(8-51)$ & $-2.099^{\dagger}$ & 0.036 \\
\hline Glasgow Outcome Scale & & $5(1-5)$ & $5(1-5)$ & $-1.677^{\dagger}$ & 0.094 \\
\hline Hemoglobin level $(\mathrm{g} / \mathrm{L})$ & & $129.50 \pm 2.12$ & $138.30 \pm 1.83$ & $-1.039^{*}$ & 0.311 \\
\hline Leukocyte count $\left(\wedge 10^{9} / \mathrm{L}\right)$ & & $8.62 \pm 3900.58$ & $9.70 \pm 3182.18$ & $-0.714^{*}$ & 0.483 \\
\hline Neutrophil count $\left({ }^{\wedge} 10^{9} / \mathrm{L}\right)$ & & $5.94 \pm 3488$ & $7.09 \pm 3849.61$ & $-0.751^{*}$ & 0.461 \\
\hline Lymphocyte count $\left({ }^{\wedge} 10^{9} / \mathrm{L}\right)$ & & $2.10 \pm 1178.65$ & $2.02 \pm 1115.91$ & $0.162^{*}$ & 0.873 \\
\hline Monocyte count $\left(\wedge 10^{9} / \mathrm{L}\right)$ & & $0.42 \pm 212.88$ & $0.42 \pm 180.62$ & $0.001^{*}$ & 0.999 \\
\hline Eosinophil count $\left(\wedge 10^{9} / \mathrm{L}\right)$ & & $0.15 \pm 111.50$ & $0.13 \pm 127.82$ & $0.416^{*}$ & 0.682 \\
\hline Basophil count $\left(\wedge 10^{9} / \mathrm{L}\right)$ & & $0.03 \pm 14.62$ & $0.05 \pm 59.37$ & $-1.147^{*}$ & 0.264 \\
\hline Platelet count $\left(\wedge 10^{9} / \mathrm{L}\right)$ & & $271.30 \pm 71870.69$ & $250.20 \pm 64813.92$ & $0.728^{*}$ & 0.475 \\
\hline Systemic inflammation response index & & $0.71(0.37-6.14)$ & $1.20(0.57-17.89)$ & $-1.116^{\dagger}$ & 0.264 \\
\hline Neutrophil to lymphocyte ratio & & $1.81(1.29-18.61)$ & $2.69(1.10-33.14)$ & $-0.992^{\dagger}$ & 0.321 \\
\hline Platelet to lymphocyte ratio & & $126.33(52.93-454.55)$ & $130.74(33.17-383.78)$ & $0.000^{\dagger}$ & 1.000 \\
\hline Lymphocyte to monocyte ratio & & $6.13(2.15-22)$ & $4.98(0.69-12.42)$ & $-0.434^{\dagger}$ & 0.664 \\
\hline Glucose (mmol/L) & & $5.72(4.44-15.26)$ & $6.66(5.05-9.44)$ & $-0.901^{\dagger}$ & 0.367 \\
\hline Sodium (mmol/L) & & $141(136-148)$ & $142(138-144)$ & $-0.438^{\dagger}$ & 0.661 \\
\hline Potassium (mmol/L) & & $4.57 \pm 0.62$ & $4.36 \pm 0.47$ & $0.892^{*}$ & 0.382 \\
\hline Creatinin $(\mu \mathrm{mol} / \mathrm{L})$ & & $62.76 \pm 0.17$ & $65.42 \pm 0.17$ & $-0.305^{*}$ & 0.763 \\
\hline Blood urea nitrogen (mmol/L) & & $10.51 \pm 7.58$ & $11.57 \pm 10.02$ & $-0.808^{*}$ & 0.428 \\
\hline Aspartate aminotransferase ( $\mu \mathrm{kat} / \mathrm{L})$ & & $0.32 \pm 7.30$ & $0.30 \pm 6.05$ & $0.326^{*}$ & 0.748 \\
\hline Alanin aminotransferase ( $\mu$ kat/L) & & $0.35(0.22-1.50)$ & $0.28(0.12-0.45)$ & $-1.243^{\dagger}$ & 0.214 \\
\hline C-reactive protein $(\mathrm{mg} / \mathrm{L})$ & & $20.20(6.90-64.80)$ & $43.90(11.10-830.00)$ & $-2.109^{\dagger}$ & 0.035 \\
\hline $\begin{array}{l}\text { Erythrocyte sedimentation } \\
\text { rate ( } \mathrm{mm} / \text { hour) }\end{array}$ & & $23.31 \pm 11.70$ & $16.00 \pm 11.20$ & $1.512^{*}$ & 0.145 \\
\hline
\end{tabular}

Abbreviations: max, maximum; min, minimum; $\mathrm{N}$, number of patients; SD, standard deviation.

${ }^{*} t$ value (Independent Samples $t$-test).

${ }^{\dagger} Z$ value (Mann-Whiney $U$ test).

${ }^{\ddagger} X^{2}$ value (Pearson'Chi-squared test). $p<0.05$.

values might be measured high, and, therefore, the shortterm prognosis of the patient might be worse, as described before by Liang et al. ${ }^{25}$ Thus, it was thought that the lymphocyte, monocyte, and eosinophil count values, PLR, LMR, SIRI, ESR, and serum AST level values might affect the short-term prognosis, indirectly. With these findings, it could be concluded that these parameters could be indirect markers in predicting the short-term prognosis of operated meningioma patients. ${ }^{24,26}$ Moreover, it was found that the patients who had high serum glucose and/or CRP values might have a longer hospital stay, so these patients might be in the older age group and therefore their short-term prognosis might be poor.
Thus, it was argued that serum CRP and glucose level values could be indirect markers in predicting shortterm prognosis. However, neither ESR nor CRP level values were correlated with any inflammatory cell count result, and the study results suggested that meningioma could not produce any cellular inflammatory response.

Although the GOS scores correlated directly with the GSC scores, platelet count values, and serum potassium level values, the likelihood-ratio test results demonstrated that monocyte count values, LMR values, and serum CRP level values could be the best parameters in predicting the short-term prognosis of meningioma patients, as described before by Bunevicius et al., Szkandera et al., and 
Table 3 Demographic findings and laboratory results of the FEMALE and MALE groups

\begin{tabular}{|c|c|c|c|c|}
\hline & FEMALE $(n=17)$ & MALE $(n=6)$ & & \\
\hline Variable & $\begin{array}{l}\text { Mean } \pm \text { SD/Median } \\
(\text { min-max)/N (\%) }\end{array}$ & $\begin{array}{l}\text { Mean } \pm \text { SD/Median } \\
(\text { min-max)/N (\%) }\end{array}$ & $t / Z$ & p-value \\
\hline Age (years old) & $60.18 \pm 13.07$ & $64.83 \pm 8.56$ & $-0.807^{*}$ & 0.429 \\
\hline Glasgow Coma Scale & $15(10-15)$ & $15(15-15)$ & $-0.859^{\dagger}$ & 0.390 \\
\hline Duration of hospital stay (days) & $9(6-31)$ & $11.50(8-51)$ & $-1.061^{\dagger}$ & 0.289 \\
\hline Glasgow Outcome Scale & $5(1-5)$ & $5(1-5)$ & $-0.194^{\dagger}$ & 0.846 \\
\hline Hemoglobin level $(\mathrm{g} / \mathrm{L})$ & $125.80 \pm 1.58$ & $154.70 \pm 1.51$ & $-3.864^{*}$ & 0.001 \\
\hline Leukocyte count $\left(\wedge 10^{9} / \mathrm{L}\right)$ & $9.51 \pm 3971.25$ & $7.89 \pm 1812.71$ & $0.952^{*}$ & 0.352 \\
\hline Neutrophil count $\left({ }^{\wedge} 10^{9} / \mathrm{L}\right)$ & $6.77 \pm 4063.86$ & $5.50 \pm 1763.23$ & $0.734^{*}$ & 0.471 \\
\hline Lymphocyte count $\left(\wedge 10^{9} / \mathrm{L}\right)$ & $2.19 \pm 1193.82$ & $1.74 \pm 918.25$ & $0.834^{*}$ & 0.414 \\
\hline Monocyte count $\left(\wedge 10^{9} / \mathrm{L}\right)$ & $0.43 \pm 189.13$ & $0.39 \pm 227.86$ & $0.374^{*}$ & 0.712 \\
\hline Eosinophil count $\left(\wedge 10^{9} / \mathrm{L}\right)$ & $0.11 \pm 100.03$ & $0.21 \pm 138.71$ & $-1.864^{*}$ & 0.076 \\
\hline Basophil count $\left({ }^{\wedge} 10^{9} / \mathrm{L}\right)$ & $0.04 \pm 44.64$ & $0.04 \pm 30.13$ & $0.090^{*}$ & 0.929 \\
\hline Platelet count $\left(\wedge 10^{9} / \mathrm{L}\right)$ & $264 \pm 77170.26$ & $257 \pm 36875.01$ & $0.216^{*}$ & 0.831 \\
\hline Systemic inflammation response index & $0.71(0.37-17.89)$ & $1.02(0.37-1.90)$ & $-1.116^{\dagger}$ & 0.264 \\
\hline Neutrophil to lymphocyte ratio & $1.81(1.10-33.14)$ & $2.73(1.72-18.61)$ & $-0.980^{\dagger}$ & 0.327 \\
\hline Platelet to lymphocyte ratio & $123.67(33.17-383.78)$ & $140.44(103.57-454.55)$ & $-0.560^{\dagger}$ & 0.575 \\
\hline Lymphocyte to monocyte ratio & $4.43(2.89-22)$ & $6.13(0.69-12.42)$ & $-0.350^{\dagger}$ & 0.726 \\
\hline Glucose (mmol/L) & $6.05(4.44-15.26)$ & $5.61(5.05-8.71)$ & $-0.772^{\dagger}$ & 0.440 \\
\hline Sodium (mmol/L) & $141(136-148)$ & $140(138-144)$ & $-0.248^{\dagger}$ & 0.805 \\
\hline Potassium (mmol/L) & $4.43 \pm 0.63$ & $4.6 \pm 04.28$ & $-0.780^{*}$ & 0.444 \\
\hline Creatinin $(\mu \mathrm{mol} / \mathrm{L})$ & $58.34 \pm 0.13$ & $78.68 \pm 0.16$ & $-3.368^{*}$ & 0.003 \\
\hline Blood urea nitrogen $(\mathrm{mmol} / \mathrm{L})$ & $10.66 \pm 9.51$ & $11.84 \pm 5.45$ & $-0.797^{*}$ & 0.434 \\
\hline Aspartate aminotransferase ( $\mu \mathrm{kat} / \mathrm{L})$ & $0.30 \pm 7.51$ & $0.34 \pm 3.15$ & $-0.708^{*}$ & 0.487 \\
\hline Alanin aminotransferase ( $\mu \mathrm{kat} / \mathrm{L})$ & $0.27(0.12-1.50)$ & $0.42(0.35-0.45)$ & $-1.754^{\dagger}$ & 0.079 \\
\hline C-reactive protein $(\mathrm{mg} / \mathrm{L})$ & $26.90(7.70-830.00)$ & $25.30(6.90-63.40)$ & $-0.280^{\dagger}$ & 0.779 \\
\hline Erythrocyte sedimentation rate (mm/hour) & $24.59 \pm 10.46$ & $7.50 \pm 2.43$ & $3.909^{*}$ & 0.001 \\
\hline
\end{tabular}

Abbreviations: max, maximum; min, minimum; $\mathrm{N}$, number of patients; SD, standard deviation.

${ }^{*} t$ value (Independent Samples $t$-test).

${ }^{\dagger} Z$ value (Mann-Whiney $U$ test). $p<0.05$

Liang et al. ${ }^{6,25}$ This finding could be interpreted as meaning that low-grade meningioma could not produce a cellular inflammatory response, but this tumor could trigger a systemic immune response, including the Th2 immune profile, and the level of this response could affect and modulate the number of circulating monocytes and lymphocytes. ${ }^{25,27}$ Furthermore, the results of the present study revealed that a low LMR value could predict poor prognosis in operated meningioma patients, as described before in the literature. ${ }^{23,25}$

In conclusion, the findings of the present study are compatible with the literature and support the information reported in the literature. However, it was recognized that in order to strongly validate the findings of the present study and to reliably predict the short-term prognosis of the operated meningioma patients, these parameters should be evaluated in a larger patient population.

\section{Limitations}

There were some limitations in the present study. First, the present study was a retrospective single-center study and, therefore, the number of patients included in the present study was low. Furthermore, the defined aims of the present study precluded adding the long-term follow-up information of patients in the present study. However, the results of the present study were very interesting. Therefore, it can be recommended that the results obtained from the present study are confirmed by further studies of larger patient groups with long-term follow-up information. Second, Kataki et al. reported that meningiomas can trigger a systemic immune response and that cell-mediated immunity shifts toward the Th2 immune profile through changes in apoptosis and necrosis. ${ }^{27}$ However, the present study results could not explore those immune responses due to its retrospective properties. Third, the comparison results of the 
preoperative and postoperative blood biochemistry findings of the patients could be included in the present study. However, because it has been well known that surgical procedures would cause dramatic changes on the blood biochemistry results; therefore, the postoperative blood biochemistry findings were not included in the present study. Finally, the radiological images and detailed histopathological evaluation reports of the patients were not evaluated and not included in the present study, because the present study aimed to define the markers for predicting the short-term prognosis in patients with meningioma using their data, obtained preoperatively.

\section{Conclusion}

At the end of the present study, correlation test results demonstrated that GCS score, platelet count value, and serum potassium level values could be predictive markers of short-term prognosis in operated meningioma patients. Additionally, these test results suggested that the lymphocyte, monocyte, and eosinophil count results, as well as PLR, ESR, serum glucose, CRP, and AST level values could predict indirectly the short-term prognosis of these patients. However, regression test results revealed surprisingly that monocyte count value, LMR value, and serum CRP level value could be the "best parameters" for prediction of the short-term prognosis. Therefore, it was recognized that all these parameters should be retested in a larger patient population for the prediction of short-term prognosis.

\section{Financial Disclosure Statements}

The authors declare that they have not engaged in any financial relationship with any company whose product might be affected by the research described or with any company that makes or markets a competing product.

\section{Informed Consent}

Since the present study had a retrospective character, the patients were informed that their information could be used in the study on the condition of protecting their personal information, and consent was obtained, so no additional consent was obtained.

\section{Conflict of Interests}

The authors have no conflict of interests to declare.

\section{References}

1 Gabrilovich DI, Ostrand-Rosenberg S, Bronte V. Coordinated regulation of myeloid cells by tumours. Nat Rev Immunol 2012; 12(04):253-268. Doi: 10.1038/nri3175

2 Mueller MM, Fusenig NE. Friends or foes - bipolar effects of the tumour stroma in cancer. Nat Rev Cancer 2004;4(11):839-849. Doi: $10.1038 /$ nrc1477

3 Diakos CI, Charles KA, McMillan DC, Clarke SJ. Cancer-related inflammation and treatment effectiveness. Lancet Oncol 2014;15 (11):e493-e503. Doi: 10.1016/S1470-2045(14)70263-3

4 Nijaguna MB, Schröder C, Patil V, et al. Definition of a serum marker panel for glioblastoma discrimination and identification of Interleukin $1 \beta$ in the microglial secretome as a novel mediator of endothelial cell survival induced by C-reactive protein. J Proteomics 2015;128:251-261. Doi: 10.1016/j.jprot.2015.07.026

5 van Alkemade $\mathrm{H}$, de Leau M, Dieleman EM, et al. Impaired survival and long-term neurological problems in benign meningioma. Neuro Oncol 2012;14(05):658-666. Doi: 10.1093/neuonc/nos013

6 Bunevicius A, Radziunas A, Tamasauskas S, et al. Prognostic role of high sensitivity C-reactive protein and interleukin-6 in glioma and meningioma patients. J Neurooncol 2018;138(02):351-358. Doi: 10.1007/s11060-018-2803-y

7 Park KJ, Kang SH, Chae YS, et al. Influence of interleukin-6 on the development of peritumoral brain edema in meningiomas. J Neurosurg 2010;112(01):73-80. Doi: 10.3171/2009.4.JNS09158

8 Strojnik T, Smigoc T, Lah TT. Prognostic value of erythrocyte sedimentation rate and $\mathrm{C}$-reactive protein in the blood of patients with glioma. Anticancer Res 2014;34(01):339-347

9 Teasdale G, Jennett B. Assessment of coma and impaired consciousness. A practical scale. Lancet 1974;2(7872):81-84

10 McMillan T, Wilson L, Ponsford J, Levin H, Teasdale G, Bond M. The Glasgow Outcome Scale - 40 years of application and refinement. Nat Rev Neurol 2016;12(08):477-485. Doi: 10.1038/ nrneurol.2016.89

11 Helmersson-Karlqvist J, Flodin M, Havelka AM, Xu XY, Larsson A. The Roche Immunoturbidimetric Albumin Method on Cobas c 501 Gives Higher Values Than the Abbott and Roche BCP Methods When Analyzing Patient Plasma Samples. J Clin Lab Anal 2016;30 (05):677-681. Doi: 10.1002/jcla.21921

12 Fogh-Andersen N, Wimberley PD, Thode J, Siggaard-Andersen O. Determination of sodium and potassium with ion-selective electrodes. Clin Chem 1984;30(03):433-436

13 Khayat Kashani HR, Azhari S, Nayebaghayee H, Salimi S, Mohammadi HR. Prediction value of preoperative findings on meningioma grading using artificial neural network. Clin Neurol Neurosurg 2020;196:105947. Doi: 10.1016/j.clineuro.2020.105947

14 Chaturvedi AK, Caporaso NE, Katki HA, et al. C-reactive protein and risk of lung cancer. J Clin Oncol 2010;28(16):2719-2726. Doi: 10.1200/JCO.2009.27.0454

15 Erlinger TP, Platz EA, Rifai N, Helzlsouer KJ. C-reactive protein and the risk of incident colorectal cancer. JAMA 2004;291(05): 585-590. Doi: 10.1001/jama.291.5.585

16 Juan H, Qijun W, Junlan L. Serum CRP protein as a differential marker in cancer. Cell Biochem Biophys 2012;64(02):89-93. Doi: 10.1007/s12013-012-9375-9

17 Colotta F, Allavena P, Sica A, Garlanda C, Mantovani A. Cancerrelated inflammation, the seventh hallmark of cancer: links to genetic instability. Carcinogenesis 2009;30(07):1073-1081. Doi: 10.1093/carcin/bgp127

18 Coussens LM, Werb Z. Inflammation and cancer. Nature 2002;420 (6917):860-867. Doi: 10.1038/nature01322

19 Heikkilä K, Ebrahim S, Lawlor DA. A systematic review of the association between circulating concentrations of $C$ reactive protein and cancer. J Epidemiol Community Health 2007;61 (09):824-833. Doi: 10.1136/jech.2006.051292

20 Cheng H, Long F, Jaiswar M, Yang L, Wang C, Zhou Z. Prognostic role of the neutrophil-to-lymphocyte ratio in pancreatic cancer: a meta-analysis. Sci Rep 2015;5:11026. Doi: 10.1038/srep11026

21 Shirai Y, Shiba H, Sakamoto T, et al. Preoperative platelet to lymphocyte ratio predicts outcome of patients with pancreatic ductal adenocarcinoma after pancreatic resection. Surgery 2015; 158(02):360-365. Doi: 10.1016/j.surg.2015.03.043

22 Stotz M, Gerger A, Eisner F, et al. Increased neutrophil-lymphocyte ratio is a poor prognostic factor in patients with primary operable and inoperable pancreatic cancer. Br J Cancer 2013;109 (02):416-421. Doi: 10.1038/bjc.2013.332

23 Szkandera J, Gerger A, Liegl-Atzwanger B, et al. The lymphocyte/ monocyte ratio predicts poor clinical outcome and improves the predictive accuracy in patients with soft tissue sarcomas. Int J Cancer 2014;135(02):362-370. Doi: 10.1002/ijc.28677 
24 Qi Q, Zhuang L, Shen Y, et al. A novel systemic inflammation response index (SIRI) for predicting the survival of patients with pancreatic cancer after chemotherapy. Cancer 2016;122(14): 2158-2167. Doi: 10.1002/cncr.30057

25 Liang RF, Li M, Li JH, Zuo MR, Yang Y, Liu YH. The significance of preoperative hematological inflammatory markers in patients with meningiomas. Clin Neurol Neurosurg 2019;182:1-4. Doi: 10.1016/j.clineuro.2019.04.020
26 Xu L, Yu S, Zhuang L, et al. Systemic inflammation response index (SIRI) predicts prognosis in hepatocellular carcinoma patients. Oncotarget 2017;8(21):34954-34960. Doi: 10.18632/oncotarget.16865

27 Kataki A, Skandami V, Memos N, et al. Similar immunity profiles in patients with meningioma and glioma tumors despite differences in the apoptosis and necrosis of circulating lymphocyte and monocyte populations. J Neurosurg Sci 2014;58(01):9-15 